\section{Campylobacter jejuni: An Emerging Foodborne Pathogen of Global Significance}

\section{Mahendra Pal ${ }^{*}$}

Narayan Consultancy on Veterinary Public Health and Microbiology, 4 Aangan, Jagnath Ganesh Dairy Road, Anand-388001, Gujarat, India

"Corresponding author: Mahendra Pal, Narayan Consultancy on Veterinary Public Health and Microbiology, 4 Aangan , Jagnath Ganesh Dairy Road, Anand-388001, Gujarat, India, E-mail: palmahendra2@gmail.com

Received date: August 16, 2017; Accepted date: September 11, 2017; Published date: September 20, 2017

Copyright: (C) $2017 \mathrm{Pal}$ M. This is an open-access article distributed under the terms of the Creative Commons Attribution License, which permits unrestricted use, distribution, and reproduction in any medium, provided the original author and source are credited.

\begin{abstract}
Emerging foodborne pathogens are significant causes of morbidity and mortality both in developed as well as developing nations. Campylobacter jejuni is an important infectious emerging foodborne bacterial zoonotic agent that causes gastrointestinal illness in humans. The infection can occur in sporadic and epidemic form. The post infections sequelae with $C$. jejuni are Guillain-Barre syndrome, reactive arthritis and irritable bowel syndrome. Globally, about one-third of Guillian-Barre syndrome cases are attributed to Campylobacter infections. It is estimated that 2.5 million cases of campylobacteriosis occur each year in the United States. The annual economic cost due to Camyplobacter associated illnesses is USA reaches up US $\$ 8$ billion. The source of infection is exogenous, and oral-fecal route is the principal mode of transmission of Campylobacter jejuni. The infection occurs through consumption of contaminated raw or undercooked meat (poultry, beef, pork, and lamb), unpasteurized milk, and untreated water. The cross-contamination from raw meat, and direct contact with infected or reservoir hosts can also be source of infection. Poultry is responsible for $50 \%-70 \%$ of human Campylobacter infections. Laboratory help is required to make an unequivocal diagnosis of disease. Most cases of Campylobacter jejuni are self-limited and generally do not require treatment. However, antimicrobial therapy with azithromycin, clarithromycin, erythromycin, chloramphanicol and gentamicin are indicated in severe cases. Certain measures such as consumption of pasteurized milk, cooked red meat and chicken, chlorinated water, application of hazard analysis critical control point in food sector, bio-security at poultry farm, sanitation in abattoir, checking cross contamination, surveillance, and health education will certainly reduce the incidence of this emerging foodborne disease. Further work on the molecular epidemiology, pathogenesis, and chemotherapy camplobacteriosis should be undertaken.
\end{abstract}

Keywords: Campylobacter jejuni; Emerging pathogen; Foods; Public health; Poultry; Zoonosis

\section{Introduction}

Recent years have witnessed the emergence of several foodborne pathogens such as Aeromonas hydrophila, Yersinia enterocolitica, Campylobacter jejuni, Listeria monocytogenese, Vibrio parahaemolyticus, Escherichia coli 0157: H7, Arcobacter butzleri, Cyclospora cayetanensis, Cryptosporidium parvum, etc. [1,2]. Among these agents, Campylobacter jejuni is one of most significant cause of bacterial diarrhea in humnas throughout the world including India [3-7]. The association of $C$. jejuni with human enteric illness was first elucidated by Butzler and co-workers in 1973. Subsequently, Skirrow in 1981 reported the etiologic role of this bacterium in enteritis of dogs and cats, and considered campylobacteriosis as a new zoonotic disease. As campylobacteriosis is not a notifiable disease, the exact data on its incidence is grossly inadequate. In the United States of America, 2.5 million people suffer annually from Campylobacter infections, of which $80 \%$ of the cases are related to ingestion of contaminated foods [8]. Among the several species of Campylobacter, $C$. jejuni is most commonly isolated from diarrheal disease in humans and animals. Other species such as $C$. jejuni ssp. doylei, $C$. coli, and $C$. lari are occasionally found to be associated with diarrhoea in human beings [7]. Campylobacter jejuni occurs as commensally in the intestinal tract of many warm blooded animals including poultry. Consumption of raw chicken, undercooked red meat, unpasteurized milk and untreated water is implicated in several outbreaks of disease [9]. The intestines of warm-blooded animals including birds act as the amplification vessel for the organisms. Manure from animals may contaminate surface water through runoff from pasture, and thus presents a risk for humans when untreated water is consumed. Furthermore, humans can be exposed to surface water through direct contact during swimming or by indirect contact through ingestion of raw products irrigated with surface water [10]. Organisms are present in feces, vaginal discharges, and the products of abortions and can be spread by direct contact, on fomites and by arthropods acting as mechanical vectors. Contaminated food and water is often the source of infections [4].

The disease is characterized by enteritis, abdominal cramps, nausea, and profuse diarrhea [11]. Guillain-Barre syndrome (GBS), reactive arthritis and irritable bowel syndrome are major complications of $C$. jejuni infections [12]. Guillain Barré syndrome, a neurological disease, can be fatal in immunocompromised populations such as AIDS patients [13]. Globally, approximately one-third of GBS cases are attributed to Campylobacter infection [12]. Laboratory techniques such as microbiological, immunological and molecular are employed to establish an unequivocal diagnosis of $C$. jejuni infection. Treatment is advised in long lasting infections with antimicrobial agents such as erythromycin, chloramphenicol, gentamicin, azithromycin, or clarithromycin [7]. As farm to fork approach is of pivotal importance in food safety, it must be observed in all food establishments to protect the health of consumers against the foodborne diseases [2]. 
In developed countries, including Australia, France, Japan, Netherlands, New Zealand, Sweden U.K. and US, Campylobacter is one of the most frequently reported causes of acute infectious diarrhea. An estimated 2.5 million cases of campylobacteriosis are recorded every year in USA [8]. Campylobacters are responsible for approximately $17 \%$ of hospitalizations resulting from food-borne infections [14]. Annual economic cost due to Camyplobacter associated illnesses is estimated up to US $\$ 8$ billion in the United States [15]. In 2004, 183,961 cases of Campylobacter infections were reported from 25 European Union countries [7].

In developing countries, direct animal-to-man transmission may be common as people live in close association with food animals; and waterborne transmission can occur due to poor sanitation [16]. The failure of routine water treatment in developed nations can cause sporadic and large outbreaks [16]. The present communication delineates the growing role of Campylobacter jejuni as an emerging foodborne pathogen of global public health importance.

\section{Etiology}

The name Campylobacter derived from the Greek word "Kampylos," which means curved. Presently, the genus Campylobacter consists of 17 species and 6 subspecies of which $C$. jejuni is most commonly reported as a cause of gastroenteritis in humans [17]. The organism is Gram-negative, non-spore-forming, microaerophilic, motile, catalase positive, oxidase positive, nitrate positive, hippurate positive, indole production negative and glucose utilization negative [7]. The bacterium can be curved, spiral, or occasionally straight rods, with size ranging from 0.2 to $0.9 \mu \mathrm{m}$ wide and 0.5 to $5 \mu \mathrm{m}$ long, and optimum growth temperature range from 35 to $45^{\circ} \mathrm{C}$ [18]. Campylobacter jejuni is quite sensitive to heat, drying, irradiation, freezing and salt concentration above $1 \%$ sodium chloride and is readily killed during pasteurization, and cooking [7].

\section{Host}

Natural infection in humans due to $C$. jejuni is reported from many countries of the world including India $[6,17]$. The organism has been isolated from baboon, cat, cattle, chicken, dog, duck, ferret, goat, horse, monkey, ostrich, pig, sheep, shellfish, turkey, and water fowl [11,17].

\section{Transmission}

Faecal-oral route is the principal mode of transmission of $C$. jejuni. Humans get the infection by consumption of unpasteurized milk, untreated water, fresh chicken, and uncooked meat of cattle, pig and sheep, which are contaminated with organisms. Close contact with diseased animals and their discharges can also transmit the infection. Clinically healthy animals may also be a source of infection [11]. It is pertinent to mention that the carcass of food animal may be contaminated during slaughtering with feces containing $C$. jejuni [17]. Swimming in natural resources of water may pose a risk to Campylobacter infection [19]. Humans may act as vectors by transferring the organism into poultry production area with contaminated clothing and foot wear [20]. Poultry is implicated in 50\%-70\% of human Campylobacter infections [21].

\section{Clinical Spectrum in Humans}

The incubation period of $C$. jejuni infection is 1-10 days with most people exhibiting clinical symptoms by 4 days [21]. The affected persons exhibit the signs of diarrhoea with mucus and blood, acute abdominal pain, fever, malaise, nausea and vomiting, headache, myalgia, arthritis, and occasionally endocarditis, meningitis, septicaemia and abortion [11,17]. The abdominal pain may persist for a week. The pain is so severe that it may be confused with appendicitis. The ileum, jejunum, colon and in few cases rectum are inflamed. The illness is most often self-limiting, as symptoms diminish after a few days up to two weeks. However, the excretion of organisms in faeces may continue for 14 to 21 days [21]. Some patients may develop Guillain-Barre syndrome, which is manifested with acute flaccid paralysis [7].

\section{Epidemiology}

The epidemiology of campylobacteriosis is still not well studied. The disease has been described from developed as well as developing nations of the world [4-7,17]. Campylobacter jejuni is the leading bacterial zoonotic pathogen, which may serious infection in children, elderly and immunocompromised patients $[7,22]$. The prevalence of $C$. jejuni infection in AIDS patients is about forty fold as compared to unaffected patients. Low levels of immunoglobulins in AIDS patients, make them sensitive to severe, persistent and recurrent $C$. jejuni infections [23]. Campylobacter jejuni and $C$. coli are the two main human gastroenteric pathogens, and are responsible for more than $95 \%$ of food-borne diseases [4]. An estimated 2.5 million cases of $C$. jejuni are diagnosed each year in the United States alone [8]. In the United Kingdom, $82 \%$ of people admitted to hospital with a diagnosis of food poisoning, were found to be suffering from Campylobacter infections [3]. The organism has been isolated from poultry, beef, pork, fish, fish products, milk, vegetables, surface water, lakes and rivers [7]. The cross contamination of Campylobacter from raw chicken to prepared food is recognized as an important risk factor. It is reported that approximately $50 \%$ of sporadic cases of Campylobacter infections in European countries are related due to handling or ingestion of chicken meat [24]. Campylobacter jejuni is also an important causative agent of traveler's diarrhea [9].

Unpasteurized milk, cheeses, butter, and yoghurt can act as vehicle of pathogen. Epps and co-investigators [21] reported that $12 \%$ of raw bovine milk samples in a dairy farm were contaminated with $C$. jejuni possibly as a result of contact with bovine faeces, contaminated water or direct contamination due to mastitis. McNaughton and co-workers [25] investigated an outbreak of Campylobacter enteritis due to ingestion of raw milk. Several studies have demonstrated the contamination of surface water such as streams, lakes and rivers with Campylobacter [26,27]. It was mentioned that $C$. jejuni was the major Campylobacter species present in water as revealed by Campylobacter species-specific PCR assays [28].

A recent study conducted on 3186 faecal samples from diarrheic patients in Kolkata, India revealed 7\% isolation rate of Campylobacter species. In this investigation, $C$. jejuni was identified as the predominant species $(78 \%)$. There was no seasonal prevalence as infection was prevalent throughout the year. Further, the recovery of $C$. jejuni was significantly was higher in children who had diarrhoea [6]. It is emphasized that growing role of $C$. jejuni should be further investigated in all cases of diarrhoea, and dysentery. It is also imperative to study the real magnitude of $C$. jejuni infections. 


\section{Diagnosis}

As campylobacteriosis simulate to other diarrheal diseases such as salmonellosis, shigellosis, colibacillosis, it is, therefore, imperative to confirm the diagnosis by employing standard laboratory tests. Direct demonstration of ' $\mathrm{S}$ ' or comma shaped organism in freshly voided stool by dark field microscopy is a simple and cheap method to make diagnosis. The pathogen can be isolated from stool, rectal swab, bile, blood and other clinical specimens on several media such as Butzler's agar, Preston medium, Skirrow medium, Campylobacter thioglycollate medium, Campylobacter cefoperazone desoxycholate agar, semi-solid Campylobacter medium, Campylobacter Blaser agar and CampyBrucella agar plate $[7,11,29]$. Immunological tests such as passive haemagglutination, complement fixation and ELISA are also useful to demonstrate antibody titer in sera samples. Currently, real time PCR is employed to screen $C$. jejuni in fecal samples [6]. Multiplex-PCR can be applied for confirmatory identification of $C$. jejuni and $C$. coli isolates, both at genus and species level [30,31]. Pulsed field gel electrophoresis (PFGE) is considered as the reference typing method for Campylobacter species [32].

\section{Treatment}

In many patients, therapy with antimicrobial drugs is usually not attempted due to mild and self-limited nature of Campylobacter infections. Nevertheless, treatment is recommended in patients who are immunocompromised or show high fever, bloody stools, and prolonged illness. Erythromycin and azithromycin are considered as the drugs of choice [7]. Some isolates of Campylobacter jejuni are susceptible to azithromycin, clarithromycin, erythromycin, chloramphanicol and gentamicin and some showed resistance to ciprofloxacin, norfloxacin, oflooxacin and nalidixic acid. Multiple resistances to two or more antibacterial antibiotics are also observed [6]. It is advised that electrolyte balance should be restored to prevent dehydration in Campylobacter enteritis.

\section{Prevention and Control}

Currently, no prophylactic vaccine is available to protect the susceptible population against $C$. jejuni infection. However, avoiding the use of untreated water, unpasteurized milk, undercooked red meat, raw poultry, hygienic handling of foods, sanitary disposal of faeces and other faeces soiled articles, biosecurity at poultry farm, good hygienic practices at meat processing plants, application of hazard analysis critical control point, training of abattoir workers, keeping children away from sick pets, active surveillance, personal hygiene and health education on basic principles of food hygiene will certainly reduce the incidence of campylobacteriosis, which has emerged as an important foodborne zoonosis of global importance $[11,17]$.

\section{Conclusion}

Microbial food pathogens still remain a major public health concern all over the world, which endangers the health of people and leads to socioeconomic costs. Campylobacter jejuni is one of the most important the causes of bacterial diarrheal disease in the world. The reservoirs of this organism include poultry, cattle, sheep, pig, and wild birds. The epidemics of Campyobacter infections are related to ingestion of raw milk, untreated water, and contact with infected animals. Multidrug resistance is frequently detected in many isolates of C. jejuni. It is imperative to pay attention to food safety from farm to table to prevent $C$. jejuni infection. Attempts should be made to develop a potent, safe and low cost vaccine to immunize the animals against a number of zoonotic foodborne pathogens including $C$. jejuni. It is emphasized to undertake further studies on the risk factors, host agent interaction and faecal carriage of bacterium in various food animals.

\section{Acknowledgements}

The author is highly indebted to Prof. Dr. R. K. Narayan for his critical comments during the preparation of the manuscript. Thanks are also due to Anubha for helping in computer work.

\section{References}

1. Pal M (2013) Public health concern due to emerging and re-emerging zoonoses. Inter J Livestock Research 3: 56-62.

2. Pal M, Mahendra R (2015) Sanitation in Food Establishments. 1st ed. LAMBERT Academic Publishing, Saarbrchen, Germany.

3. Adak G, Cowden J, Nicholas S, Evans H (1995) The public health laboratory service national case-control study of primary indigenous sporadic cases of Campylobacter infection. Epidemiol Infect 115: 15-397.

4. Allos BM (2001) Campylobacter jejuni infections: update on emerging issues and trends. Clin Infect Dis 32: 1201-1206.

5. Coker AO, Isokpehi RD, Thomas BN, Amisu KO, Obi CL (2002) Human campylobacteriosis in developing countries. Emerg Infect Dis 8: 237-243.

6. Mukherjee S, Babitzke P, Kearns DB (2013) FliW and FliS function independently to control cytoplasmic flagellin levels in Bacillus subtilis. J Bacteriol 195: 297-306.

7. Pal M (2014) Impact of emerging bacterial foodborne pathogens on human health. Ph.D. Lecture Notes. Addis Ababa University, College of Veterinary Medicine, Debre Zeit, Ethiopia. pp.1-15.

8. Bhaduri S, Cottrell B (2004) Survival of cold stressed Campylobacter jejuni on ground chicken and chicken skin during frozen state. App Environ Microbiol 70:7103-7109.

9. Hadush A, Pal M (2013) Detection of Camylobacter from foods and its epidemiology. J Pub Health Epidemiol 5: 357-361.

10. Wagenaar JA, French NP, Havelaar AH (2013) Preventing Campylobacter at the source: Why is it so difficult? Clin Infect Dis 57: 1600-1606.

11. Pal M (2007) Zoonoses. 2nd ed. Satyam Publishers, Jaipur, India.

12. WHO (2012) The global view of campylobacteriosis: report of an expert consultation, Utrecht, Netherlands, 9-11 July 2012.

13. Mishu B, Blaser MJ (1993) Role of infection due to Campylobacter jejuni in the initiation of Guillain-Barré Syndrome. Clin Infect Dis 17: 104-108.

14. Mead PS, Slutsker L, Dietz V, McCaig LF, Bresee JS, et al. (1999) Foodrelated illness and death in the United States. Emerg Infect Dis. 5: 607-625.

15. Buzby JC, Allos BM, Roberts T (1997) The economic burden of Campylobacter associated Guillain-Barré syndrome. J Infect Dis 176: 192-197.

16. Suzuki H, Yamamoto S (2009) Campylobacter contamination in retail poultry meats and by-products in Japan: A literature Survey of Food Control. J Food Cont 20: 531-537.

17. WHO (2016) Campylobacter Fact Sheet. World Health Organization, Geneva, Switzerland.

18. Cox NA, Richardson LJ, Musgrove MT (2010) Campylobacter jejuni and other Campylobacters. Juneja VK and Sofos JN (Edit). Pathogens and Toxins in Foods Challenges and Interventions. ASM Press, Washington, DC.

19. Schonberg-Norio D, Takkinen J, Hanninen ML, Katila ML, Kaukoranta SS, et al. (2004) Swimming and Campylobacter infections. Emerging Infect Dis 10: 1474-1477.

20. Doyle P, Beuchat R (2007) Food Microbiology. Fundamentals and Frontiers 3rd Edition. Washington DC. ASM Press pp. 817-836. 
Citation: Pal M (2017) Campylobacter jejuni: An Emerging Foodborne Pathogen of Global Significance. J Exp Food Chem 3: 130. doi: 10.4172/2472-0542.1000130

Page 4 of 4

21. Epps SV, Harve RB, Hum ME, Phillip TD, Anderso RC, et al. (2013) Foodborne Campylobacter: Infections, metabolism, pathogenesis and reservoirs. Int J Environ Res Public Health 10: 6292-6304.

22. Humphrey TJ, O'Brien S, Madsen M (2007) Campylobacters as zoonotic pathogens: A food production perspective. Int J Food Microbiol 117: 237-257.

23. Barros-Velázquez J, Jiménez A, Villa TG (1999) Isolation and typing methods for the epidemiologic investigation of thermotolerant campylobacters. Internat Microbiol 2: 217-226.

24. Jacobs-Reitsma WF (1997) Aspects of epidemiology of Campylobacter in poultry. Vet Quarterly 19: 113-117.

25. McNaughton RD, Leyland R, Mueller L (1982) Outbreak of Campylobacter enteritis due to consumption of raw milk. Can Med Assoc J 126: 657-658.

26. Eyles R, Niyogi D, Townsend C, Benwell G, Weinstein P (2003) Spatial and temporal patterns of Campylobacter contamination underlying public health risk in the Taieri River, New Zealand. J Environ Qual 32: 1820-1828.

27. Denis M, Tanguy M, Chidaine B, Laisney MJ, Me'graud F, et al. (2011) Description and sources of contamination by Campylobacter spp. of river water destined for human consumption in Brittany, France. Pathologie Biologie 59: 256-263.
28. Lu J, Ryu H, Vogel J, Domingo JS, Ashbolta NJ (2013) Molecular detection of Campylobacter spp. and fecal indicator bacteria during the northern migration of sandhill cranes (Grus canadensis) at the Central Platte River. Appl Environ Microbiol 79: 3762-3769.

29. Jeffrey JS, Hunter A, Atwill ER (2000) A field-suitable, semisolid aerobic enrichment medium for isolation of Campylobacter jejuni in small numbers. J Clin Microbiol 38: 1668-1669.

30. Linton D, Lawson AJ, Owen RJ, Stanley J (1997) PCR detection, identification to species level, and fingerprinting of Campylobacter jejuni and Campylobacter coli direct from diarrheic samples. J Clin Microb 35: 2568-2572.

31. Denis M, Soumet C, Rivoal K, Ermel G, Blivet D, et al. (1999) Development of a m-PCR for simultaneous identification of Campylobacter jejuni and Campylobacter coli. Lett Appl Microbiol 29: 406-410.

32. Behringer M, Miller WG, Oyarzabal OA (2011) Typing of Campylobacter jejuni and Campylobacter coli isolated from live broilers and retail broiler meat by flaA-RFLP, MLST, PFGE and REP-PCR. J Microbiol Methods 84: 194-201. 